\title{
Obstructions aux déformations de représentations galoisiennes réductibles et groupes de classes
}

\author{
par ARIANE MÉZARD
}

Avec toute mon amitié pour George Gras

RÉSUMÉ. Nous développons une nouvelle stratégie pour comprendre la nature des obstructions aux déformations d'une représentation galoisienne globale $\bar{\rho}$ réductible, impaire de dimension 2 . Ces obstructions s'interprètent en termes de groupe de Šafarevič. D'après [BöMé], elles sont reliées à des conjecture arithmétiques classiques (Conjecture de Vandiver, conjecture de Greenberg). Dans cet article, nous introduisons un autre groupe de Šafarevič associé au corps $L$ fixe par ker $\bar{\rho}$. Nous comparons les deux groupes en prenant les co-invaraints par $\operatorname{Im} \bar{\rho}$. Cette stratégie conduit à de nouvelles conditions d'annulation des obstructions en termes de groupes des classes de $L$.

\begin{abstract}
In this paper, we develop a new strategy to understand the obstructions to deformations of reducible odd 2-dimensional global Galois representations $\bar{\rho}$. It is known that these obstructions are localized in a Šafarevič group. After [BöMé] these obstructions are related with several classical conjectures (Vandiver's conjecture, Greenberg's conjecture). The idea of this note is to introduce another Šafarevič group depending on the field $L$ fixed by $\operatorname{ker} \bar{\rho}$. We then compare the two groups by taking the co-invariant by $\operatorname{Im} \bar{\rho}$. This strategy yields new conditions for the vanishing of the obstructions in terms of class groups of $L$.
\end{abstract}

\section{Introduction}

Soit $p$ un nombre premier impair. Soit $\bar{\rho}: G_{S}(E) \rightarrow \mathrm{Gl}_{2}\left(\mathbf{F}_{p}\right)$ une représentation galoisienne impaire réductible non ramifiée en dehors d'un ensemble fini de places $S_{E}$ d'un corps totalement réel $E$. Nous supposons que $S_{E}$ contient les places au-dessus de $p$ et les places archimédiennes. Le groupe $G_{S}(E)=\operatorname{Gal}\left(E_{S} / E\right)$ est le groupe de Galois de la plus grande extension $E_{S}$ de $E$ non ramifiée en dehors de $S_{E}$. La représentation adjointe de $\bar{\rho}$ est notée ad $\bar{\rho}$. Lorsque que ad $\bar{\rho}^{G_{S}(E)}=\mathbf{F}_{p}$ Id, Mazur a montré

Manuscrit reçu le 25 mai 2004.

Mots clefs. Anneau de déformation versel, groupe de classes, groupe de Šafarevič. 
l'existence d'un anneau de déformations universel $R_{G_{S}}(\bar{\rho})$ qui paramètre toutes les déformations de $\bar{\rho}([\mathrm{Ma}])$. La structure de cet anneau $R_{G_{S}}(\bar{\rho})$ est intimement liée aux groupes de cohomologie $H^{i}\left(G_{S}(E), \operatorname{ad} \bar{\rho}\right), i=1,2$. Nous souhaitons déterminer les informations arithmétiques contenues dans ces groupes de cohomologie. D'après [BöMé], l'absence d'obstruction de certaines déformations est liée à la nullité de certaines parties du groupe des $S_{E}$-classes de $E$. Ce résultat a notamment conduit à un nouveau critère de véracité de la conjecture de Vandiver ([BöMé], Proposition 3.3.1). La stratégie de [BöMé] repose sur un dévissage de la représentation adjointe comme $F_{p}[H]$-module pour

$$
H \cong\left(\begin{array}{ll}
* & 0 \\
0 & *
\end{array}\right)
$$

correspondant à la partie d'ordre premier à $p$ de $\operatorname{Im} \bar{\rho}$.

L'objet de cette note est une étude plus précise du lien entre l'arithmétique du corps $E$ et les déformations de $\bar{\rho}$. Ici, nous trivialisons l'action du groupe de Galois. Nous travaillons au niveau du corps $L$ fixé par $\operatorname{ker} \bar{\rho}$. Nous redescendons au corps $E$ en prenant les co-invariants par $\operatorname{Im} \bar{\rho}$. Il s'agit donc de déterminer l'influence de la partie d'ordre $p$ de $\operatorname{Im} \bar{\rho}$,

$$
U \cong\left(\begin{array}{ll}
1 & * \\
0 & 1
\end{array}\right)
$$

Les méthodes et les résultats sont différents et précisent ceux de [BöMé] car les $\mathbf{F}_{p}[U]$-modules ne sont pas semi-simples, contrairement aux $\mathbf{F}_{p}[H]$ modules.

Nous commençons par rappeler brièvement les informations arithmétiques du corps $E$ pertinentes pour la connaissance de l'espace tangent aux déformations $H^{1}\left(G_{S}(E), \operatorname{ad} \bar{\rho}\right)(\S 3)$. Puis nous étudions le groupe d'obstruction $H^{2}\left(G_{S}(E), \operatorname{ad} \bar{\rho}\right)(\S 4)$. Nous obtenons un nouveau critère qui précise sous quelles hypothèses nous pouvons calculer explicitement l'anneau universel de déformations (Théorème 4.1). Il s'agit d'annuler certaines parties du quotient $\mathrm{CL}_{S}(L)$ du groupe des $p$-classes de $L$ par les classes des idéaux premiers correspondant aux places finies de $L$ au-dessus de $S$. Nous discutons enfin ces hypothèses $(\S 4.2)$ et les comparons aux résultats déjà obtenus.

L'auteur remercie Thong Nguyen Quang Do pour de nombreuses discussions sur la théorie d'Iwasawa et le rapporteur dont les remarques ont permis de simplifier la rédaction de cet article.

\section{Notation}

Soit $\bar{\rho}: G_{S}(E) \rightarrow \mathrm{Gl}_{2}\left(\mathbf{F}_{p}\right)$ une représentation galoisienne impaire réductible non ramifiée en dehors d'un ensemble fini de places $S_{E}$ d'un corps 
totalement réel $E$ contenant les places au-dessus de $p$ et les places archimédiennes. Soit $G_{S}(E)=\operatorname{Gal}\left(E_{S} / E\right)$ le groupe de Galois de la plus grande extension $E_{S}$ de $E$ non ramifiée en dehors de $S_{E}$. Notons $H$ (resp. $U$ ) la partie d'ordre premier à $p$ (resp. d'ordre $p$ ) de $J \cong \operatorname{Im} \bar{\rho}, L$ le corps fixe par ker $\bar{\rho}$ et $F$ la sous-extension de $E$ dans $L$ laissée fixe par $U$. Résumons les notations à l'aide du diagramme suivant :

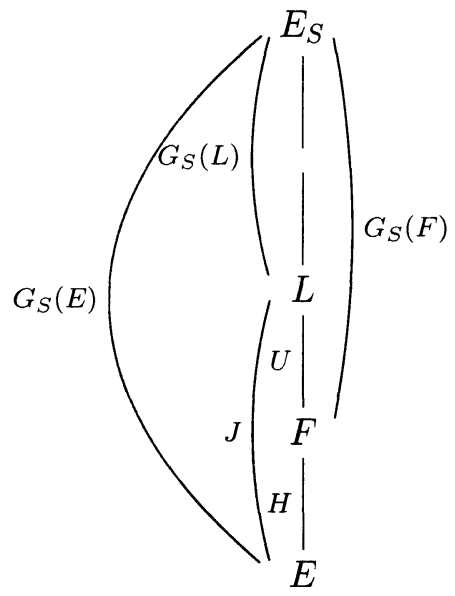

où $G_{S}(L)=\operatorname{Gal}\left(E_{S} / L\right)$ et $G_{S}(F)=\operatorname{Gal}\left(E_{S} / F\right)$. Pour alléger les discussions, nous supposons que $\mu_{p} \subset L$ (donc $\mu_{p} \subset F$ car $\operatorname{Gal}(L / K)$ est d'ordre premier à $p)$. Cette hypothèse n'est pas essentielle, il suffit de travailler avec $L\left(\mu_{p}\right)$. Pour $v \in S_{K}$, notons $K_{v}$ le complété de $K$ à la place $v$ et $G_{K_{v}}=\operatorname{Gal}\left(\overline{K_{v}} / K_{v}\right)$. Le quotient du groupe des $p$-classes de $K$ par les classes d'idéaux premiers correspondant aux places finies de $S_{K}$ est noté $\mathrm{Cl}_{S}(K)$. Soit $M$ un $G_{S}(E)$-module fini. Définissons les groupes de Šafarevič

$$
\amalg_{S}^{i}(K, M)=\operatorname{ker}\left(H^{i}\left(G_{S}(K), M\right) \rightarrow \coprod_{v \in S_{K}} H^{i}\left(G_{K_{v}}, M\right)\right), \quad i=1,2 .
$$

Le module $M$ tordu par le caractère cyclotomique est noté $M(1)$. Le dual de Pontryagin de $M$ est $M^{*}=\operatorname{Hom}_{\mathbf{Z}_{p}}\left(M, \mathbf{Q}_{p} / \mathbf{Z}_{p}\right)$. Notons $h^{i}\left(G_{S}(E), M\right)=$ $\operatorname{dim}_{\mathbf{F}_{p}} H^{i}\left(G_{S}(E), M\right)$ pour $i=0,1,2$.

Soit $\widehat{H}$ le groupe des caractères de $H=\operatorname{Gal}(F / E)$, i.e des homomorphismes de $H$ dans $\mathbf{F}_{p}^{*}$. Si $\varphi \in \widehat{H}$ est un caractère de $H$, définissons $e_{\varphi}$ comme l'idempotent associé dans $\mathbf{Z}_{p}[H]$. Cet idempotent existe car $p$ est premier avec l'ordre de $H$. Pour un $\mathbf{Z}_{p}[H]$-module $M$, notons $M_{\varphi}=e_{\varphi} M$. Définissons $\mathbf{F}_{p}^{\varphi}$ (resp. $\mathbf{Z}_{p}^{\varphi}$ ) comme le $\mathbf{F}_{p}[H]$-module $\mathbf{F}_{p}$ (resp. le $\mathbf{Z}_{p}[H]$ module $\mathbf{Z}_{p}$ ) avec l'action de $H$ donnée par $\varphi$. Soit $M^{\varphi}=M \otimes \mathbf{z}_{p} \mathbf{Z}_{p}^{\varphi}$.

Nous rappelons également la décomposition de $\operatorname{ad} \bar{\rho}$ en tant que $\mathbf{F}_{p}\left[G_{S}(E)\right]$-module ([BöMé] Example 4.1.4). L'action de $G_{S}(E)$ est 
triviale sur les matrices scalaires, donc comme $\mathbf{F}_{p}\left[G_{S}(E)\right]$-module

$$
\operatorname{ad} \bar{\rho}=\operatorname{ad}^{0} \bar{\rho} \oplus \mathbf{F}_{p}
$$

où $\operatorname{ad}^{0} \bar{\rho}$ est le $\mathbf{F}_{p}\left[G_{S}(E)\right]$-module des matrices de trace nulle. De plus nous avons une filtration de $\operatorname{ad}^{0} \bar{\rho}$ comme $\mathbf{F}_{p}\left[G_{S}(E)\right]$-module définit par les deux suites exactes suivantes

$$
\begin{aligned}
& 0 \rightarrow \mathbf{F}_{p}^{\psi^{-1}} \rightarrow W \rightarrow \mathbf{F}_{p} \rightarrow 0 \\
& 0 \rightarrow W \rightarrow \operatorname{ad}^{0} \bar{\rho} \rightarrow \mathbf{F}_{p}^{\psi} \rightarrow 0
\end{aligned}
$$

pour $W \cong\left\{\left(\begin{array}{ll}a & b \\ 0 & -a\end{array}\right), a, b \in \mathbf{F}_{p}\right\}, \bar{\rho}=\left(\begin{array}{ll}\chi_{1} & * \\ 0 & \chi_{2}\end{array}\right)$ et $\psi=\chi_{1}^{-1} \chi_{2}$. Notons enfin $\mathscr{V}=\left\{\right.$ triv, $\left.\psi, \psi^{-1}\right\}$ et $M_{\gamma^{\prime}}=M_{\text {triv }} \oplus M_{\psi} \oplus M_{\psi^{-1}}$ pour $M$ un $\mathbf{Z}_{p}[H]$-module.

\section{Description de l'espace tangent}

La structure de l'anneau universel de déformations

$$
R_{G_{S}}(\bar{\rho})=W(k)\left[\left[T_{1}, \ldots, T_{d}\right]\right] / I
$$

est liée aux groupes de cohomologies $H^{i}\left(G_{S}(E), \operatorname{ad} \bar{\rho}\right) i=1,2$ : en particulier, le nombre minimal de générateurs de $R_{G_{S}}(\bar{\rho})$ est

$$
d=\operatorname{dim}_{\mathbf{F}_{p}} H^{1}\left(G_{S}(E), \operatorname{ad} \bar{\rho}\right)
$$

et le nombre minimal de relations est inférieur à $\operatorname{dim}_{\mathbf{F}_{p}} H^{2}\left(G_{S}(E), \operatorname{ad} \bar{\rho}\right)$. Dans le cas des représentations modérées (dont l'image est d'ordre premier à $p$ ), Boston et Ullom ont calculé ces dimensions ([BoUl]). Pour les représentations réductibles (de type Borel), le résultat suivant permet de restreindre notre étude au 2-groupe de cohomologie $H^{2}\left(G_{S}(E)\right.$, ad $\left.\bar{\rho}\right)$.

Lemme 3.1. Soit $\chi(E, M)=\sum_{i=0}^{2}(-1)^{i} h^{i}\left(G_{S}(E), M\right)$ la caractéristique d'Euler-Poincaré d'un $G_{S}(E)$-module fini $M$. Nous avons

$$
\chi(E, \operatorname{ad} \bar{\rho})=2 \chi\left(E, \mathbf{F}_{p}\right)+\chi\left(E, \mathbf{F}_{p}^{\psi}\right)+\chi\left(E, \mathbf{F}_{p}^{\psi^{-1}}\right)
$$

Preuve : Il suffit d'écrire les deux suites exactes longues de cohomologie associées à la décomposition (1),(2) de ad $\bar{\rho}$.

Soit

$\delta=2 h^{2}\left(G_{S}(E), \mathbf{F}_{p}\right)+h^{2}\left(G_{S}(E), \mathbf{F}_{p}^{\psi}\right)+h^{2}\left(G_{S}(E), \mathbf{F}_{p}^{\psi^{-1}}\right)-h^{2}\left(G_{S}(E), \operatorname{ad} \bar{\rho}\right)$.

Alors

$$
\begin{aligned}
h^{1}\left(G_{S}(E), \operatorname{ad} \bar{\rho}\right)= & 2 h^{1}\left(G_{S}(E), \mathbf{F}_{p}\right)+h^{1}\left(G_{S}(E), \mathbf{F}_{p}^{\psi}\right) \\
& +h^{1}\left(G_{S}(E), \mathbf{F}_{p}^{\psi^{-1}}\right)-1+\delta .
\end{aligned}
$$


Soit $\varphi \in\left\{\operatorname{triv}, \psi, \psi^{-1}\right\}$. Comme $H=\operatorname{Gal}(F / E)$ est d'ordre premier à $p$ et $G_{S}(F)$ agit trivialement sur $\mathbf{F}_{p}^{\varphi}$, alors

$$
H^{1}\left(G_{S}(E), \mathbf{F}_{p}^{\varphi}\right) \cong H^{1}\left(G_{S}(F), \mathbf{F}_{p}^{\varphi}\right)^{H} \cong \operatorname{Hom}\left(G_{S}(F), \mathbf{F}_{p}^{\varphi}\right)^{H}
$$

Soit $P_{S}(F)$ le groupe de Galois de la plus grande pro- $p$-extension de $F$ non ramifiée en dehors de $S$. Soit

$$
\overline{P_{S}(F)}=P_{S}(F) /\left[P_{S}(F), P_{S}(F)\right] P_{S}(F)^{p}
$$

son quotient de Frattini. Donc

$$
H^{1}\left(G_{S}(E), \mathbf{F}_{p}^{\varphi}\right) \cong \operatorname{Hom}\left(\overline{P_{S}(F)}, \mathbf{F}_{p}^{\varphi}\right)^{H}
$$

En utilisant les résultats de [Ko], Boston et Ullom ont donné un présentation commode de $\mathbf{F}_{p}[H]$-module $\overline{P_{S}(F)}$ ([BoUl] Proposition 3.2) :

$$
\overline{P_{S}(F)} \cong \mathbf{F}_{p} \oplus \operatorname{Ind}_{H_{\infty}}^{H} \widetilde{\mathbf{F}_{p}} \oplus \operatorname{Coker}\left(\mu_{p}(F) \rightarrow \oplus_{v \in S(F)} \mu_{p}\left(F_{v}\right)\right) \oplus \amalg_{S}^{2}\left(F, \mathbf{F}_{p}\right)^{*}
$$

où $H_{\infty}$ est un sous-groupe d'ordre deux engendré par une conjugaison complexe de $H$ et $\widetilde{\mathbf{F}_{p}}$ est le $\mathbf{F}_{p}\left[H_{\infty}\right]$-module non-trivial. Cette présentation (1) permet de calculer $h^{1}\left(G_{S}(E), \mathbf{F}_{p}^{\varphi}\right)$. En effet il suffit de calculer le produit intérieur des $\mathbf{F}_{p}[H]$-modules $\left(\overline{P_{S}(F)}, \mathbf{F}_{p}^{\varphi}\right)$ (voir Proposition 4.2 [BoUl]). Pour déterminer $\delta$ et $h^{1}\left(G_{S}(E)\right.$, ad $\left.\bar{\rho}\right)$, il nous suffit donc de calculer la dimension $\mathrm{du}$ groupe d'obstruction $H^{2}\left(G_{S}(E)\right.$, ad $\left.\bar{\rho}\right)$. En revanche, pour déterminer l'idéal $I$ des relations de $R_{G_{S}}(\bar{\rho})$, la dimension de $H^{2}\left(G_{S}(E), \operatorname{ad} \bar{\rho}\right)$ ne suffit pas. Il faut connaître la structure de ce groupe d'obstruction.

\section{Description du groupe d'obstruction}

D'après [BöMé], il est possible de déterminer explicitement l'anneau universel de déformations $R_{G_{S}}(\bar{\rho})$, pourvu que la condition de non obstruction globale soit satisfaite :

$$
\amalg_{S}^{2}(E, \operatorname{ad} \bar{\rho})=0
$$

En effet, d'après la suite de Poitou-Tate

$$
\begin{aligned}
& 0 \rightarrow \amalg_{S}^{2}(E, \operatorname{ad} \bar{\rho}) \rightarrow H^{2}\left(G_{S}(E), \operatorname{ad} \bar{\rho}\right) \rightarrow \\
& \coprod_{v \in S_{E}} H^{2}\left(G_{E_{v}}, \operatorname{ad} \bar{\rho}\right) \rightarrow H^{0}\left(G_{S}(E), \operatorname{ad} \bar{\rho}^{*}(1)\right)^{*} \rightarrow 0
\end{aligned}
$$

le groupe $H^{2}\left(G_{S}(E)\right.$, ad $\left.\bar{\rho}\right)$ est connu pourvu que la condition (1) soit satisfaite. Pour étudier la condition de non-obstruction globale (1) nous commençons par introduire la condition de non-obstruction globale au niveau $L$ :

$$
\amalg_{S}^{2}(L, \operatorname{ad} \bar{\rho})_{J}=0
$$


i.e nullité du groupe des éléments de $\amalg_{S}^{2}(L, \operatorname{ad} \bar{\rho})$ co-invariants par $J=\operatorname{Im} \bar{\rho}$. Par dévissage de ad $\bar{\rho}$, nous obtenons un critère pour que cette condition (2) soit satisfaite (Proposition 4.1). Nous constatons ensuite que la nonobstruction globale au niveau $L$ implique la non-obstruction globale (Proposition 4.2). Nous discutons de la réciproque (Théorème 4.2).

\subsection{Déformations globalement non obstruées.}

Lemme 4.1. Notons $J=\operatorname{Im} \bar{\rho}$. Les deux suites suivantes sont exactes

$$
\begin{gathered}
\amalg_{S}^{2}\left(L, \mathbf{F}_{p}^{\psi}\right)_{J} \rightarrow \amalg_{S}^{2}(L, W)_{J} \rightarrow \amalg_{S}^{2}\left(L, \mathbf{F}_{p}\right)_{J} \rightarrow 0, \\
\amalg_{S}^{2}(L, W)_{J} \rightarrow \amalg_{S}^{2}\left(L, \operatorname{ad}^{0} \bar{\rho}\right)_{J} \rightarrow \amalg_{S}^{2}\left(L, \mathbf{F}_{p}^{\psi^{-1}}\right)_{J} \rightarrow 0 .
\end{gathered}
$$

Preuve : Rappelons la décomposition du $G_{S}(E)-\operatorname{module} \operatorname{ad}^{0} \bar{\rho}$ :

$$
\begin{gathered}
0 \rightarrow \mathbf{F}_{p}^{\psi} \rightarrow W \rightarrow \mathbf{F}_{p} \rightarrow 0, \\
0 \rightarrow W \rightarrow \operatorname{ad}^{0} \bar{\rho} \rightarrow \mathbf{F}_{p}^{\psi^{-1}} \rightarrow 0 .
\end{gathered}
$$

Ces deux suites sont des suites de $G_{S}(L)$-modules triviaux. Donc elles sont scindées comme suites de $G_{S}(L)$-modules. Prenons leurs images par le foncteur exact $\amalg_{S}^{2}(L, \cdot)$ puis par le foncteur "co-invariant par $J$ " qui est exact à gauche. Nous obtenons alors le résultat annoncé.

Lemme 4.2. Nous avons $\amalg_{S}^{2}\left(L, \mathbf{F}_{p}^{\varphi}\right)_{J}=0$ si et seulement si $\mathrm{Cl}_{S}(L)_{\varphi^{-1} \omega}=$ 0 .

Preuve : D'après la dualité de Poitou-Tate

$$
\amalg_{S}^{2}\left(L, \mathbf{F}_{p}^{\varphi}\right)_{J} \cong\left(\varpi_{S}^{1}\left(L, \mathbf{F}_{p}^{\varphi^{-1} \omega}\right)^{J}\right)^{*} .
$$

Or

$$
\amalg_{S}^{1}\left(L, \mathbf{F}_{p}^{\varphi^{-1} \omega}\right)^{U}=\operatorname{Hom}_{U}\left(\mathrm{Cl}_{S}(L), \mathbf{F}_{p}^{\varphi^{-1} \omega}\right)=\operatorname{Hom}\left(\mathrm{Cl}_{S}(L)_{U}, \mathbf{F}_{p}\right)^{\varphi^{-1} \omega} .
$$

Donc, vu que $H$ est d'ordre premier à $p$,

$$
\left(\amalg_{S}^{1}\left(L, \mathbf{F}_{p}^{\varphi^{1} \omega}\right)^{J}\right)^{*} \cong\left(\mathrm{Cl}_{S}(L)_{U} /(p)\right)_{H}^{\varphi \omega^{-1}} \cong\left(\mathrm{Cl}_{S}(L)_{U} /(p)\right)_{\varphi^{-1} \omega} .
$$

Enfin comme $\mathrm{Cl}_{S}(L)$ est un $p$-groupe, nous avons

$$
\left(\mathrm{Cl}_{S}(L)_{U} /(p)\right)_{\varphi^{-1} \omega}=0 \Longleftrightarrow\left(\mathrm{Cl}_{S}(L)_{\varphi^{-1} \omega}\right)_{U}=0 \Longleftrightarrow \mathrm{Cl}_{S}(L)_{\varphi^{-1} \omega}=0 .
$$

D'où le lemme. 
Rappelons les notations $\mathscr{V}=\left\{\operatorname{Id}, \psi, \psi^{-1}\right\}, J=\operatorname{Im} \bar{\rho}$. Comme ad $\bar{\rho}=$ $\operatorname{ad}^{0} \bar{\rho} \oplus \mathbf{F}_{p}$, les lemmes 4.2, 4.1 conduisent au résultat suivant :

Proposition 4.1. Si $\mathrm{Cl}_{S}(L)_{\gamma^{\prime} \omega}=0$ alors $\amalg_{S}^{2}(L, \operatorname{ad} \bar{\rho})_{J}=0$.

La proposition 4.1 montre que la condition

$$
\mathrm{Cl}_{S}(L)_{\omega \gamma^{\prime}}=0
$$

implique la non-obstruction globale au niveau $L$. Il reste maintenant à comparer la non-obstruction globale et la non-obstruction globale au niveau $L$, autrement dit à rapprocher les groupes de Šafarevič $\amalg_{S}^{2}\left(L, \operatorname{ad}^{0} \bar{\rho}\right)_{J}$ et $\amalg_{S}^{2}\left(E, \operatorname{ad}^{0} \bar{\rho}\right)$.

Proposition 4.2. Supposons qu'il existe une place $v_{0}$ de $F$ telle que $U_{v_{0}} \cong$ $U$. Alors pour tout $\mathbf{F}_{p}\left[G_{S}(E)\right]$-module $M$,

$$
\amalg_{S}^{2}(L, M)_{U} \rightarrow \amalg_{S}^{2}(F, M) \quad \text { et } \amalg_{S}^{2}(L, M)_{J} \rightarrow \amalg_{S}^{2}(E, M) .
$$

PREUVE : Nous avons le diagramme composé de suites exactes suivant :

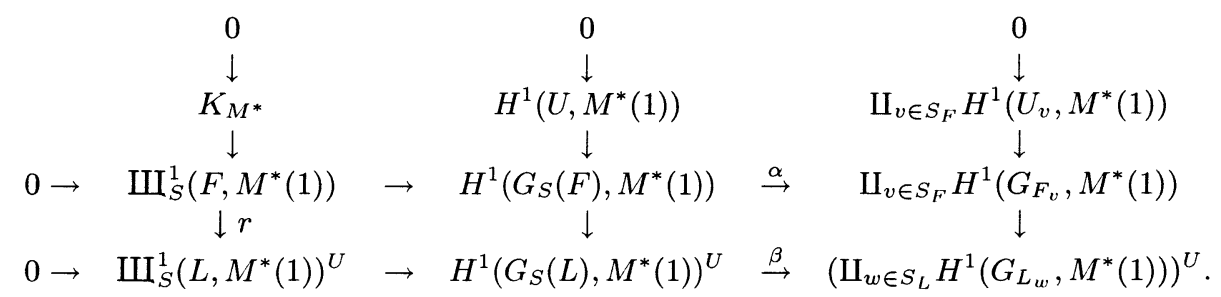

Notons $s: \operatorname{Im} \alpha \rightarrow \operatorname{Im} \beta$. Le lemme du serpent nous donne

$$
0 \rightarrow K_{M^{*}} \rightarrow H^{1}\left(U, M^{*}(1)\right) \rightarrow \operatorname{ker} s .
$$

Or ker $s \subset \amalg_{v \in S_{F}} H^{1}\left(U_{v}, M^{*}(1)\right)$ et par hypothèse

$$
H^{1}\left(U, M^{*}(1)\right) \hookrightarrow \amalg_{v \in S_{F}} H^{1}\left(U_{v}, M^{*}(1)\right),
$$

d'où $K_{M^{*}}=0$ et l'injection

$$
\amalg_{S}^{1}\left(F, M^{*}(1)\right) \hookrightarrow \amalg_{S}^{1}\left(L, M^{*}(1)\right)^{U} .
$$

La dualité de Poitou-Tate (nous avons supposé que $\mu_{p} \subset F$ ) et l'exactitude du foncteur "invariant par $H$ " permettent alors d'obtenir le résultat annoncé.

Remarque. Cette preuve donne également une description du conoyau de l'injection $r: \amalg_{S}^{1}\left(F, M^{*}(1)\right) \rightarrow \amalg_{S}^{1}\left(L, M^{*}(1)\right)^{U}:$

$$
\text { Coker } r=\operatorname{Coker}\left(H^{1}\left(U, M^{*}(1)\right) \rightarrow \operatorname{Im} \alpha \cap \amalg_{v \in S_{F}} H^{1}\left(U_{v}, M^{*}(1)\right)\right) .
$$

En effet, en ajoutant au diagramme de la preuve de la proposition 4.2, une ligne (correspondant à l'inflation-restriction pour les colonnes) nous 
obtenons Coker $s \subset H^{2}(U, M)$ et d'après la suite de Poitou-tate $H^{2}(U, M) \hookrightarrow \amalg_{v \in S_{F}} H^{2}\left(U_{v}, M\right)$.

En rassemblant les résultats des propositions 4.1 et 4.2 , nous obtenons un nouveau critère pour que les déformations de $\bar{\rho}$ soient globalement non obstruées :

Théorème 4.1. Supposons qu'il existe une place $v_{0}$ de $F$ telle que $U_{v_{0}} \cong$ $U$. Si $\mathrm{Cl}_{S}(L)_{\Upsilon^{\prime} \omega}=0$, alors les déformations de $\bar{\rho}$ sont globalement non obstruées.

D'après la remarque 4.1 , nous pouvons discuter de la réciproque $\mathrm{du}$ Théorème 4.1, car nous contrôlons le conoyau de

$$
\amalg_{S}^{1}\left(F, \operatorname{ad} \bar{\rho}^{*}(1)\right) \hookrightarrow \amalg_{S}^{1}\left(L, \operatorname{ad} \bar{\rho}^{*}(1)\right)^{U}
$$

et donc le noyau de

$$
\amalg_{S}^{2}(L, \operatorname{ad} \bar{\rho})_{J} \rightarrow \amalg_{S}^{2}(E, \operatorname{ad} \bar{\rho}) .
$$

Nous reprenons plus en détail cette discussion au paragraphe $\S 4.2$ (voir Théorème 4.2).

Les hypothèses du théorème 4.1 sont plus naturelles que l'hypothèse de bonne filtration du critère obtenu dans [BöMé] (Corollaire 4.1.8), dont nous rappelons l'énoncé:

Proposition 4.3. Supposons que la filtration (1), (2) de $\operatorname{ad}^{0} \bar{\rho}$ soit bonne, i.e pour tout $v \in S_{E}, \mu_{p}\left(E_{v}\right)=\{1\}$ et il existe $v_{0} \in S_{E}$ tel que $p$ divise l'ordre $\left|\bar{\rho}\left(G_{E_{v_{0}}}\right)\right|, U_{v_{0}} \cong U,\left.\psi\right|_{G_{E_{v_{0}}}}=\left.\omega^{-1}\right|_{G_{E_{v_{0}}}}$ et les ordres de $\omega$ comme caractère de $G_{E_{v_{0}}}$ et comme caractère de $G_{S}(E)$ sont les mêmes.

Si $\mathrm{Cl}_{S}(F)_{y^{\prime} \omega}=0$ et si la filtration de $\operatorname{ad}^{0} \bar{\rho}$ est bonne pour $S$, alors le problème de déformations est globalement non-obstrué.

Dans le paragraphe suivant nous comparons les hypothèses $\mathrm{Cl}_{S}(F)_{\omega \%}=$ 0 et $\mathrm{Cl}_{S}(L)_{\omega \mathcal{Y}^{\prime}}=0$. Remarquons que ces deux critères ne proposent que des conditions suffisantes de non-obstruction globale; il serait bien sûr intéressant de trouver un exemple de problème de déformations globalement non obstrué qui mette en défaut l'un des deux critères.

A ce stade, nous pouvons résumer les implications logiques par le diagramme suivant,

$$
\begin{gathered}
\left\{\mathrm{Cl}_{S}(L)_{\omega y^{\prime}}=0\right\} \Longrightarrow\left\{\begin{array}{l}
\text { non-obtruction globale } \\
\text { au niveau } L
\end{array}\right\} \\
\qquad .1 \| 4.1 \\
\left.\Downarrow \mathrm{Cl}_{S}(F)_{\omega y^{\prime}}=0\right\} \stackrel{4.3}{\Longrightarrow}\left\{\begin{array}{l}
\text { non-obtruction globale } \\
\text { au niveau } F
\end{array}\right\} .
\end{gathered}
$$


Les références indiquées sur les flèches renvoient aux hypothèses de la proposition 4.3 et du théorème 4.1 .

4.2. Conditions de non-obstruction globale. Nous allons maintenant étudier le noyau du morphisme de la proposition 4.2

$$
\amalg_{S}^{2}(L, M)_{J} \rightarrow \amalg_{S}^{2}(E, M) .
$$

Nous commençons par établir un nouveau lien entre les relations de l'anneau de déformations universel et les relations du groupe de Galois $G_{S}(E)$ :

Lemme 4.3. Soit $M$ un $G_{S}(E)$-module de p-torsion. Nous avons

$$
H^{2}\left(G_{S}(L), M\right)_{J} \stackrel{\sim}{\longrightarrow} H^{2}\left(G_{S}(E), M\right)
$$

Preuve : D'après [Se] $\S 3.5$ Proposition 17 , le groupe profini $G_{S}(E)$ de dimension cohomologique $\operatorname{cd}_{p} G_{S}(E)=2$, admet un unique module dualisant noté $D$, tel que pour tout $G_{S}(E)$-module $M$ de $p$-torsion,

$$
H^{2}\left(G_{S}(E), M\right)^{*} \cong \operatorname{Hom}(M, D)^{G_{S}(E)} \text {. }
$$

D'après [Se] $§ 3.5$ Proposition 18, le sous-groupe ouvert $G_{S}(L)$ de $G_{S}(E)$ admet également $D$ comme module dualisant. Par conséquent

$$
\begin{aligned}
\left(H^{2}\left(G_{S}(L), M\right)^{*}\right)^{J} & \cong\left(\operatorname{Hom}(M, D)^{G_{S}(L)}\right)^{J} \\
& \cong \operatorname{Hom}(M, D)^{G_{S}(E)} \\
& \cong H^{2}\left(G_{S}(E), M\right)^{*}
\end{aligned}
$$

D'après le lemme 4.3 , nous avons

$$
H^{2}\left(G_{S}(L), \operatorname{ad} \bar{\rho}\right)_{J} \cong H^{2}\left(G_{S}(E), \operatorname{ad} \bar{\rho}\right)
$$

avec action triviale de $G_{S}(L)$ sur ad $\bar{\rho}$. Cet isomorphisme est un lien entre les relations de $G_{S}(L)$ (décrite par $H^{2}\left(G_{S}(L), \mathbf{F}_{p}\right)$ ) et les relations de $R_{G_{S}}(\bar{\rho})$. Par ailleurs le théorème 4.3 permet également de comparer les conditions

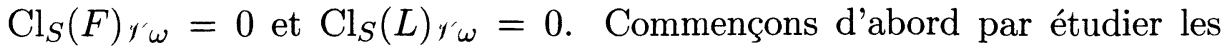
co-invariants de certains modules semi-locaux.

Pour $M$ un $G_{S}(F)$-module de $p$-torsion, nous notons

$$
\widetilde{\oplus}_{w \in S_{L}} H^{2}\left(G_{L_{w}}, M\right)=\operatorname{ker}\left(\coprod_{w \in S_{L}} H^{2}\left(G_{L_{w}}, M\right) \rightarrow H^{0}\left(G_{S}(L), M^{*}(1)\right)^{*}\right) .
$$

Et nous définissons de façon analogue $\widetilde{\oplus}_{v \in S_{F}} H^{2}\left(G_{F_{v}}, M\right)$. Nous avons alors un morphisme induit

$$
t:\left(\widetilde{\oplus}_{w \in S_{L}} H^{2}\left(G_{L_{w}}, M\right)\right)_{U} \rightarrow \widetilde{\oplus}_{v \in S_{F}} H^{2}\left(G_{F_{v}}, M\right) .
$$


Théorème 4.2. Soit $M$ un $G_{S}(F)$-module de p-torsion.

i. Le morphisme induit

$$
t:\left(\widetilde{\oplus}_{w \in S_{L}} H^{2}\left(G_{L_{w}}, M\right)\right)_{U} \rightarrow \widetilde{\oplus}_{v \in S_{F}} H^{2}\left(G_{F_{v}}, M\right)
$$

est surjectif.

ii. Si de plus l'action de $G_{S}(L)$ est trivial sur $M$ et s'il existe une place $v_{0}$ de $F$ telle que $U_{v_{0}} \cong U$, alors $t$ est un isomorphisme et la suite suivante est exacte :

$$
\left(\widetilde{\oplus}_{w \in S_{L}} H^{2}\left(G_{L_{w}}, M\right)\right)^{J} \rightarrow \amalg_{S}^{2}(L, M)_{J} \rightarrow \amalg_{S}^{2}(E, M) \rightarrow 0 .
$$

Preuve : i. Nous avons le diagramme composé de suites exactes :

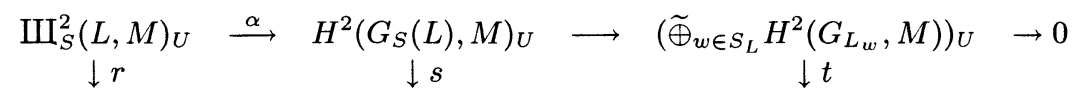

$$
\begin{aligned}
& 0 \rightarrow \amalg_{S}^{2}(F, M) \stackrel{\beta}{\longrightarrow} H^{2}\left(G_{S}(F), M\right) \quad \stackrel{\gamma}{\longrightarrow} \tilde{\oplus}_{v \in S_{F}} H^{2}\left(G_{F_{v}}, M\right) \quad \rightarrow 0 .
\end{aligned}
$$

où $s$ est un isomorphisme d'après le lemme 4.3. D'après le lemme du serpent ([We] $§ 1.3 .2$ ), nous avons la suite exacte

(3) $0 \rightarrow \operatorname{ker} \alpha \rightarrow \operatorname{ker} r \rightarrow \operatorname{ker} s \rightarrow \operatorname{ker} t \rightarrow$

$$
\text { coker } r \rightarrow \text { coker } s \rightarrow \text { coker } t \rightarrow \operatorname{coker} \gamma \rightarrow 0 .
$$

D'où coker $t=0$ et la surjectivité de

$$
t:\left(\widetilde{\oplus}_{w \in S_{L}} H^{2}\left(G_{L_{w}}, M\right)\right)_{U} \rightarrow \widetilde{\oplus}_{v \in S_{F}} H^{2}\left(G_{F_{v}}, M\right) .
$$

ii. Supposons que $M$ est un $G_{s}(L)$-module trivial et qu'il existe une place $v_{0}$ de $F$ telle que $U_{v_{0}} \cong U$. D'après la proposition 4.2 , coker $r=0$. Ainsi d'après (3) ker $t=0$ et $t$ est un isomorphisme. De la suite exacte

$$
0 \rightarrow \amalg_{S}^{2}(L, M) \rightarrow H^{2}\left(G_{S}(L), M\right) \rightarrow \widetilde{\oplus}_{w \in S_{L}} H^{2}\left(G_{L_{w}}, M\right) \rightarrow 0,
$$

on obtient la suite exacte

$$
\begin{aligned}
\left(\widetilde{\oplus}_{w \in S_{L}} H^{2}\left(G_{L_{w}}, M\right)\right)^{U} & \rightarrow \amalg_{S}^{2}(L, M)_{U} \rightarrow \\
H^{2}\left(G_{S}(L), M\right)_{U} & \rightarrow\left(\widetilde{\oplus}_{w \in S_{L}} H^{2}\left(G_{L_{w}}, M\right)\right)_{U} \rightarrow 0 .
\end{aligned}
$$

Puis en appliquant le foncteur "invariant par $H$ " (qui est exact car $H$ est d'ordre premier à $p$ ),

$$
\begin{aligned}
\left(\widetilde{\oplus}_{w \in S_{L}} H^{2}\left(G_{L_{w}}, M\right)\right)^{J} & \rightarrow \amalg_{S}^{2}(L, M)_{J} \rightarrow \\
& H^{2}\left(G_{S}(L), M\right)_{J} \rightarrow\left(\widetilde{\oplus}_{w \in S_{L}} H^{2}\left(G_{L_{w}}, M\right)\right)_{J} \rightarrow 0 .
\end{aligned}
$$

Enfin d'après $(3)(\operatorname{ker} \alpha)^{H} \cong(\operatorname{ker} r)^{H}$, d'où ii. 
Avec des hypothèses de décomposition de places de $S_{E}$ dans $F$, nous obtenons l'équivalence suivante :

Corollaire 4.1. Supposons qu'il existe $v_{0} \in S_{F}$ tel que $U_{v_{0}} \cong U$ et que pour tout $v \in S_{E}$, le caractère $\varphi^{-1} \omega \in \widehat{H}$ induit un caractère non trivial $\left(\varphi^{-1} \omega\right)_{v}$ de $\widehat{H_{v}}$. Alors,

$$
\mathrm{Cl}_{S}(F)_{\varphi^{-1} \omega}=0 \Longleftrightarrow \mathrm{Cl}_{S}(L)_{\varphi^{-1} \omega}=0
$$

PREUVE : D'après le théorème 4.2, nous avons la suite exacte suivante :

$$
0 \rightarrow \Delta^{\prime} \rightarrow \amalg_{S}^{2}\left(L, \mathbf{F}_{p}^{\varphi}\right)_{J} \rightarrow \amalg_{S}^{2}\left(E, \mathbf{F}_{p}^{\varphi}\right) \rightarrow 0
$$

avec

$$
\Delta^{\prime}=\operatorname{Im}\left(\left(\widetilde{\oplus}_{w \in S_{L}} H^{2}\left(G_{L_{w}}, \mathbf{F}_{p}^{\varphi}\right)\right)^{J} \rightarrow \amalg_{S}^{2}\left(L, \mathbf{F}_{p}^{\varphi}\right)_{J}\right)
$$

Or

$$
\begin{aligned}
& \left(\widetilde{\oplus}_{w \in S_{L}} H^{2}\left(G_{L_{w}}, \mathbf{F}_{p}^{\varphi}\right)\right)^{J}= \\
& \quad \operatorname{ker}\left(\left(\amalg_{w \in S_{L}} H^{2}\left(G_{L_{w}}, \mathbf{F}_{p}^{\varphi}\right)\right)^{J} \rightarrow\left(H^{0}\left(G_{S}(L), \mathbf{F}_{p}^{\varphi^{-1} \omega}\right)^{*}\right)^{J}\right)
\end{aligned}
$$

et

$$
\left(\amalg_{w \in S_{L}} H^{2}\left(G_{L_{w}}, \mathbf{F}_{p}^{\varphi}\right)\right)^{J} \cong \amalg_{v \in S_{E}}\left(\mathbf{F}_{p}^{\varphi \omega^{-1}}\right)^{H_{v}} \cong \amalg_{v \in S_{E}}\left(\mathbf{F}_{p}\right)_{\left(\varphi^{-1} \omega\right)_{v}} .
$$

Ainsi d'après les hypothèses sur $\left(\varphi^{-1} \omega\right)_{v}$,

$$
\left(\amalg_{w \in S_{L}} H^{2}\left(G_{L_{w}}, \mathbf{F}_{p}^{\varphi}\right)\right)^{J} \cong 0 .
$$

Donc

$$
\Delta^{\prime} \cong 0 \quad \text { et } \quad \amalg_{S}^{2}\left(L, \mathbf{F}_{p}^{\varphi}\right)_{J} \cong \amalg_{S}^{2}\left(E, \mathbf{F}_{p}^{\varphi}\right) .
$$

Le lemme 4.2 permet alors de conclure.

Avec des hypothèses de non-décomposition des places de $S_{E}$ dans $F$, nous pouvons alors comparer nos deux critères de non-obstruction globale (Théorème 4.1 et Proposition 4.3):

Corollaire 4.2. Supposons que pour tout $v \in S_{E}, \mu_{p}\left(E_{v}\right)=\{1\}$, qu'il existe $v_{0} \in S_{F}$ tel que $U_{v_{0}} \cong U$ et que toutes les places de $S_{E}$ ne sont pas décomposées dans $F$. Alors pour $\psi \notin\left\{\omega, \omega^{-1}\right\}$,

$$
\mathrm{Cl}_{S}(F)_{\mathcal{Y}^{\prime} \omega}=0 \Longleftrightarrow \mathrm{Cl}_{S}(L)_{\mathcal{Y}^{\prime} \omega}=0 \text {. }
$$

L'étude des déformations de représentations galoisiennes conduit donc à une nouvelle approche des questions de nullité de certains groupes de classes. Ces questions sont au centre de nombreuses conjectures en arithmétique (conjecture de Vandiver, conjecture de Greenberg...). Ces conjectures ont également été étudiées grâce à des méthodes issues de la théorie d'Arakelov ([So]). Il serait interessant de confronter ces deux approches. 


\section{Bibliographie}

[BöMé] G. BÖCKLE, A. MÉzARD, The prime-to-adjoint principle and unobstructed Galois deformations in the Borel case. J. Number Theory 78 (1999), 167-203.

[BoUl] N. Boston, S.V. Ullom, Representations related to CM elliptic curves. Math. Proc. Phil. Soc. 113 (1993), 71-85.

[Ko] H. Косн, Galoische Theorie der p-Erweiterung. Mathematische Monographien. VEB Deutscher Verlag der Wissenschaften 10, 1970.

[Ma] B. MAZUR, Deforming Galois representations. Dans Galois groups over Q, Y. Ihara, K. Ribet, J.-P. Serre eds, MSRI Publ. 16, (1987), 385-437.

[Se] J.-P. SERRE, Cohomologie galoisienne. Lecture Notes in Math. 5, 1973.

[So] C. SoulÉ, Perfect forms and the Vandiver conjecture. J. Reine Angew. Math. 517 (1999), 209-221.

[Ta] J.T. TATE, Global class field theory. Dans Algebraic number theory. J. Cassels A. Fröhlich eds, Academic Press 1967, 163-203.

[We] C. WeIBEL, An introduction to homological algebra. Cambridge University press 1994.

Ariane MÉzARD

Laboratoire de mathématiques, UMR 8628

Université de Paris-Sud XI

91405 Orsay Cedex, France

E-mail : ariane.mezard@math.u-psud.fr

$U R L:$ http://www.math.u-psud.fr/ mezard/ 